\title{
Los estudiantes universitarios y el aspecto socio- económico
}

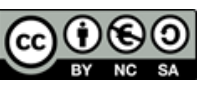

\section{University students and the socio-economic aspect}

Yolanda Tatiana Carrasco Ruano. ${ }^{1}$

Recibido: 14-03-2018 / Revisado: 16-03-2018 / Aceptado: 22-03-2018 / Publicado: 05-04-2018

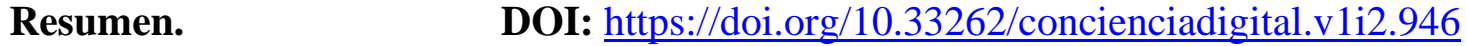

Uno de los principales Factores que tenemos los estudiantes universitarios es el factor económico la falta de recursos que dificultan para continuar con los estudios superiores. A pesar que son muchos los factores que obstaculizan a los estudiantes; siendo el más importante el socio-económico muchas de las veces los mismos estudiantes tienen que generar sus propios recursos para solventar sus carreras; porqué los padres de familia no tienen recursos suficientes o no cuentan con un trabajo estable para solventar los estudios de sus hijos y a veces con un numeroso núcleo familiar. Es por este factor qué los universitarios no pueden desarrollarse al cien por ciento y mejor obstan por abandonar sus carreras En la actualidad la educación se ha convertido en algo imprescindible para poder desarrollarse tanto intelectualmente, y como progreso de la sociedad ser una mejor persona, si la mayoría de los jóvenes estudiarán sería algo muy fundamental porqué ayudaría a disminuir la pobreza en las familias ya que tendrían más oportunidades de trabajar y también contribuirá para la sociedad con sus conocimientos adquiridos durante su preparación universitaria. En poco recurso que genera el estado para la educación superior no es suficiente para solventar; muchos gastos que genera la universidad. El presente trabajo consiste en analizar y sacar conclusiones de los factores más determinantes para no poder seguir con los estudios superiores.

Palabras claves: Estudiante, Educación superior, aspecto socio-económico

\footnotetext{
${ }^{1}$ Ciencia digital, Ambato, Ecuador, tcarrascor@yahoo.com
} 


\section{Abstract.}

One of the main factors that we have university students is the economic factor lack of resources that make it difficult to continue with higher education. Although there are many factors that hinder students; being the most important the socio-economic many of the times the same students have to generate their own resources to solve their careers; because parents do not have enough resources or do not have a stable job to pay for their children's studies and sometimes with a large family nucleus. It is by this factor that the university students can not develop to the one hundred percent and better obstan by abandoning their careers At present the education has become something indispensable to be able to develop so much intellectually, and as progress of the society to be a better person, if most of the young people will study would be very fundamental because it would help to reduce poverty in the families since they would have more opportunities to work and also contribute to society with their knowledge acquired during their university preparation. In little resource generated by the state for higher education is not enough to solve; many expenses generated by the university. The present work consists of analyzing and drawing conclusions from the most determining factors in order to be able to continue with higher studies.

Keywords: Student, Higher education, socio-economic aspect.

\section{Introducción.}

\section{¿Qué es el Aspecto Socio-económico?}

Avalos, D. (2017), Navarro, Definición ABC, (2015) y Definiciones-de.com, (2016) mencionan que el aspecto socio económico es la capacidad de un grupo de individuos o sociedad a manejar la tolerancia entre ellos y sus niveles de economía. La sociedad y la economía van de la mano depende tanto la economía de la sociedad para ser sustentable es así como la sociedad depende de la economía para poder progresar. En la actualidad este tema es tan relevante ya que hay naciones donde la corrupción y la ambición de poder han opacado la libre soberanía en la economía y la sociedad se ha visto truncada si ningún merecimiento de lo que netamente les pretense. Es así que la economía mundial ha decaído y la sociedad ha dado millones de protestas para que los gobernantes tomen cartas en el asunto. Menciona que son dos términos fusionados que son el aspecto de la realidad de social y económica en la sociedad en conjunto. El nivel económico se entiende que es la capacidad social y económica que abarca una familia o país, esto se distinguirá del tipo de familia, el tipo de hogar, el número de individuos que conforma el núcleo familiar, la sociedad que les rodea, el ingreso económico y las oportunidades de trabajo que se presenta para los jefes de ese hogar. Aquí entra diferentes ciencias que quieren llegar a un mismo fin cuales son los factores principales para que una población mantenga una sociedad unida y una economía sustentable. En donde podemos darnos cuenta de la situación económica y social de las 
personas donde existe la mayor discriminación por estos factores es allí donde se mide los niveles de jerarquía de cada persona empezando desde sus condiciones de vida hasta sus ingresos y nivel de educación de cada individuo.

\section{Materiales y Métodos}

Llegue a la conclusión de que hablar del aspecto socio económico es la fusión entre las relaciones que mantienen la sociedad y la economía para poder subsistir una con la otra, también que son la mayoría de gobernantes que pueden modificar drásticamente la economía en un país o nación. También que el aspecto socio económico rige las necesidades que puede tener un núcleo familiar y que sin una economía sustentable ese núcleo familia puede quebrantarse y hasta desaparecer es muy alarmante ver en los países subdesarrollados los cuales no cuentan con una economía sustentable afectando a los núcleos familiares y a la sociedad en general. Menciona que la principal tarea del aspecto socio-económico es darse cuenta de las condones de vida de un cierto sector de cualquier país para medir el grado de estudio alfabetismo empleo tomando todas las medidas necesarias para generar todas las facilidades que un estado facilite todas las posibilidades de sobresalir a un estudiante lo cual generaría más personas preparadas con mejores oportunidades de sobresalir ante la sociedad y que su familia pueda tener una vida sustentable.

Tabla N 1 Factores por los cuales estudiantes universitarios truncan su carrera

\begin{tabular}{|l|r|r|r|}
\hline Razón x la que no asiste & $\mathbf{2 0 1 2}$ & $\mathbf{2 0 1 3}$ & $\mathbf{2 0 1 4}$ \\
\hline Edad & $1,59 \%$ & $1,15 \%$ & $0,02 \%$ \\
\hline Terminó sus estudios & $0,20 \%$ & $0,63 \%$ & $0,29 \%$ \\
\hline Falta recursos económicos & $35,58 \%$ & $31,35 \%$ & $24,49 \%$ \\
\hline Fracaso escolar & $3,51 \%$ & $5,01 \%$ & $4,26 \%$ \\
\hline Por trabajo & $16,64 \%$ & $9,65 \%$ & $8,50 \%$ \\
\hline Temor maestros & $0,19 \%$ & $0,77 \%$ & \\
\hline Por asistir nivelación SENESCYT & & & $2,44 \%$ \\
\hline Enfermedad o discapacidad & $9,92 \%$ & $12,46 \%$ & $10,74 \%$ \\
\hline Quehaceres del hogar & $5,29 \%$ & $6,26 \%$ & $9,52 \%$ \\
\hline Familia no permite & $1,24 \%$ & $1,49 \%$ & $1,83 \%$ \\
\hline No hay establecimientos educativos & $0,98 \%$ & $2,24 \%$ & $1,38 \%$ \\
\hline No está interesado & $11,60 \%$ & $15,54 \%$ & $18,31 \%$ \\
\hline Por embarazo & $1,68 \%$ & $2,32 \%$ & $2,88 \%$ \\
\hline Por falta de cupo & $3,23 \%$ & $3,26 \%$ & $4,27 \%$ \\
\hline Otra razón & $8,35 \%$ & $7,88 \%$ & $11,05 \%$ \\
\hline \hline
\end{tabular}

Fuente:https://educacion.gob.ec/wp-content/uploads/downloads/2016/01

/Publicaciones/PUB_EstadisticaEducativaVol1_mar2015.pdf

Esta imagen muestra los principales factores por los cuales unos estudiantes universitarios truncaban su carrera y optaba por trabajar u otras ocupaciones del día a día para poder subsistir y ayudar en su hogar. Se ve que la principal causa que dejan de estudiar es por la falta de una economía sustentable, la segunda es por trabajar y muy pocos logran llevar a la par las dos actividades, el trabajar y estudiar es un esfuerzo cual no muchos muchachos puedes sostener en un ritmo constante. También existen centros educativos encaminados con 
la educación, allí tiene que cumplir con todos los requisitos requeridos donde establecen normas y reglas durante el periodo educativa iniciando un conocimiento básico de las distintas ramas de su carrera y posteriormente llegando a obtener todos los conocimientos necesarios para llegar hacer un buen profesional en el área de su especialización.

\section{Estudiante y el aspecto socio-económico}

Venemedia, M. (2018), Porto, S. (2008) y Ucha, U. (2012) mencionan que un estudiantes es aquella persona que aprende nuevos conocimientos a lo largo de su carrera estudiantil ya sea en primaria, secundaria o universidad. Las instituciones educativas ya sean academias, escuelas o colegios son el centro donde se desenvuelven la mayor parte de estudiantes. El que estudia pone en práctica sus conocimientos sobre lo aprendido para poder ser un estudiante lo pueden hacer de varias formas ya sea con sus propios recursos puedes contratar a una persona experta en la materia de la cual deseas aprender estudiar de forma particular, esta es una manera autónoma de estudiar. También existen centros educativos encaminados con la educación, allí tiene que cumplir con todos los requisitos requeridos donde establecen normas y reglas durante el periodo educativa iniciando un conocimiento básico de las distintas ramas de su carrera y posteriormente llegando a obtener todos los conocimientos necesarios para llegar hacer un buen profesional en el área de su especialización.

Figura $\mathbf{N}$ 1: porcentajes de estudiantes universitarios que optaron por trabajar (hombres y mujeres).

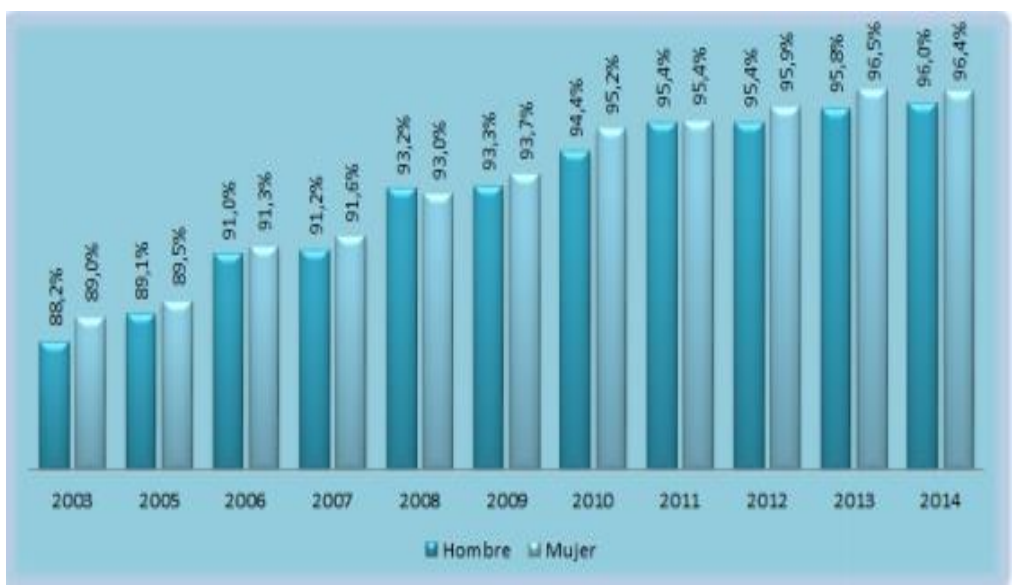

Fuente:https://educacion.gob.ec/wp-content/uploads/downloads/2016/01/

Publicaciones/PUB_EstadisticaEducativaVol1_mar2015.pdf

Resultados: Como podemos observar en el grafico muestra que los porcentajes van en aumento cada año y las cifras de los señores universitarios que abandonan sus estudios es solo por el hecho de generar una economía sustentable para su hogar y solo un número mínimo opta por seguir estudiando, aquellos que se sacrifican mucho más que un estudiantes promedio. Lo cual ha dado un crecimiento poblacional de estudiantes también que el 
porcentaje de personas que trabajan y estudian se ha elevado y los jóvenes universitarios optan por seguir trabajando y no solo estudiar deja a parte.

\section{La Educación superior y la falta de plazas de trabajo para estudiantes.}

Navarro, G. (2015), Ministerio de educación Republica de Colombia, (2009) y Naciones unidas, (2017). Menciona que la educación superior se da en dos niveles: pregrado y postgrado. Define que es un progreso de educación permanente el cual conlleva a los estudiantes a ser más perspicaces y susceptibles a opinión y criticidad, mejorado sus virtudes y aflorando sus mejores conceptos de la carrera que decidió seguir o lo que le apasiona. La educación trata de un derecho de toda persona y un servicio público que el estado debe regular, reformar y ejecutar la inspección y vigilancia de cada centro educativo y universidad. La educación superior es la formación de nuevos profesionales en los ámbitos laborales. Hoy en día es muy preocupante tener o conseguir un trabajo por las pocas fuentes de trabajo que existe en el sector laboral por ende los señores estudiantes no goza de un trabajo estable. Las plazas de trabajo en la actualidad destinada a los universitarios no ha incrementado y solo ha generado preocupación ya que algunas carreras universitarios son muy caras y los hogares no puedes gestionar ni colaborar al estudiantes a seguir y muchos optan por abandonar y muy pocos solo trabajan y sustentan sus estudios. Los cual genera aspectos socio dependiente que se simplifica en dos simples palabras vagancia y parasitismo, el ser parasito de la sociedad y del círculo familiar es quitar los apoyos que se le pueden dar a otra persona que si pueda llevar y sostener sus estudios al pie de la letra.

En resumen, la educación superior se da en dos niveles el pregrado y el postgrado, también que es la continuidad de la educación primaria y secundaria, también es la que regirá y forjara su carácter a una persona. La educación superior no está accesible para todas las naciones y mucho menos para todas las clases sociales y las mujeres, es por eso que la ONU tiene como objetivo para el 2030 globalizarla educación superior porque con ella lograremos dar un cambio drástico en la sociedad y también porque con estudios realizados pudieron notar que la educación superior mejora la calidad de vida de cada individuo. Cabe recalcar que las plazas de trabajo en el Ecuador son mínimas por ende el factor socio-económico se ha convertido en unos de principales problemas de los estudiantes que estudian la educación superior. 
Figura N 2. Las divisiones económicas del Ecuador.

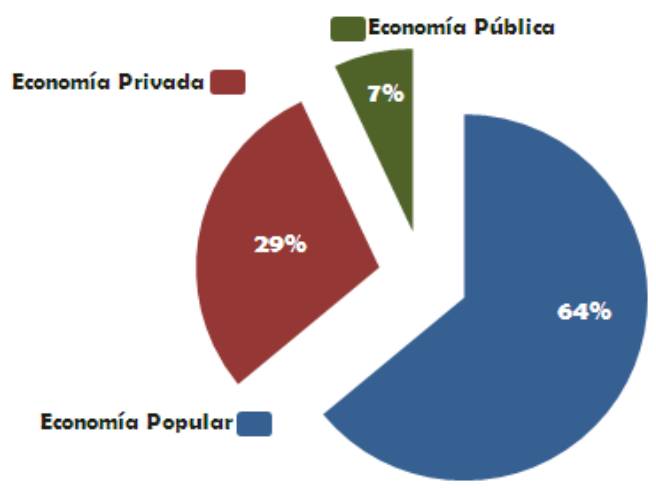

Fuente: https://ericfochoa.wordpress.com/2012/01/27/economia-popular-y-empleoen-ecuador/

\section{Resultado}

Se puede denotar que las economías del país se dan en tres ámbitos son (la economía popular, la economía pública y la economía popular) cabe recalcar que la economía popular es la más alta que genera empleo pero este empleo es mal remunerado y sobre explotado algunas veces. Es decir que la economía del pueblo es la que abarca más espacio pero menos riquezas lo cual por parte de los estados y las grandes fábricas es cómo podemos modificar la economía de un país si uno de ellos truncan sus actividades económicas afectaría más a la economía popular y hay afectaría más a los universitarios que dependen de su trabajo para acabar sus estudios.

\section{Los Estudiantes Universitarios y el aspecto socio-económico}

Quintana, F. (2017) y Alvarenga Valiente, M. (2014) mencionan que en la actualidad la economía ha dado diversos factores para que en muchos países estén perjudicados por la disminución de plazas de trabajo para las familias, es ahí donde la educación en muchos de los hogares es truncada por la falta de un ingreso económico sustentable, cuando hablamos de estudiantes nos referimos a todos desde el nivel más bajo como el kínder hasta el más alto las universidades. En el caso de las universidades los señores estudiantes por diversos factores no han podido culminar sus estudios y han optado por dejar de estudiar y dedicarse a llevar el pan para su hogar al no contar con una economía sustentable y nadie quien los pueda apoyar económicamente en sus estudios, porque las ganas las han de tener. 


\section{Conclusiones}

- En conclusión puedo decir que el factor socio-económico actualmente es el que más afecta a la sociedad en general como los estudiantes universitarios también les afectan y algunos optan por abandonar sus estudios y muy pocos en conseguir un trabajo y sustentarse ellos mismos y esto les generan muchos inconvenientes como por ejemplo las malas notas también problemas psicológicos y dependencia de algunos vicios como son el alcoholismo, tabaquismo y consumo de sustancias sujetas a fiscalización. Por ende el apoyo de los familiares y mucho más del gobierno se lograra que el ecuador salga de los países tercermundistas y entre en las vías al desarrollo con gente más preparada y decidida a triunfar con muchas más oportunidades.

- En mucho de los casos los estudiantes al no constar con un apoyo económico optan por conseguir un trabajo y en muchos de los casos ese trabajo no es muy bien remunerado, llevando a estudiante a una sobre explotación tanto física como mental los cual genera un problema socio dependiente y a pagar a compañeros para que les hagan los deberes y ellos no aprenden nada generándoles problemas en los estudios y en los hogares. La satisfacciones un hogar es ver a uno de sus familiares acabar su carrera universitaria porque así saben que tendrás más oportunidades de mejorar su economía.

- La economía en el país ha decaído gracias a los extranjeros provenientes de Venezuela han hecho que la economía baje y la economía de algunos hogares sufran un descenso y lo cual afecta también a los estudiantes de cada familia. Por qué no se pueden desempeñar al cien por ciento en todas sus facultades, es decir que si la economía del país generaría fuga de cerebros lo cual aria que ese país no pueda avanzar socialmente y mucho menos tecnológicamente.

- Es esencial que se generen fuentes de trabajo por parte del ministerio para que los jóvenes universitarios puedan mejorar su economía y desempeñarse más bien en el ámbito estudiantil, las plazas de trabajo en la actualidad no dan con el tiempo libre que un joven universitario tiene y si algún gobierno entrante propusiera nuevas plazas de trabajo para ayudar a la economía de los estudiantes esto sería un apoyo muy 
factible, también ayudaría a ganar experiencia a los futuros profesionales en cada una de las carreras

\section{Referencias bibliográficas.}

Alvarenga Valiente, A. J. (11 de febrero de 2014). UES.EDU.COM. Recuperado el 29 de 10 de 2018, de Aspecto socioeconimoco: http://ri.ues.edu.sv/6381/1/Incidencia\%20del\%20factor\%20socioecon\%C3\%B3mic o\%20en\%20e1\%20rendimiento\%20acad\%C3\%A9mico\%20de\%20losas\%20estudia ntes\%20de\%20la\%20Carrera\%20de\%20Licenciatura\%20en\%20Ciencias\%20de\%2 0la\%20Educaci\%C3\%B3n\%20de\%20la\%20Universidad\%20de

Avalos, R. (12 de 01 de 2017). UMAK wiki. Recuperado el 29 de 10 de 2018, de aspecto socioeconomico: https://wiki.umaic.org/wiki/Socioecon\%C3\%B3mico

Definiciones-de.com. (24 de 08 de 2016). definiciones.de, 1. Recuperado el 29 de 10 de 2018, de definicionde $\quad$ socioeconomico: https://www.definicionesde.com/Definicion/de/socioeconomico.php

Ministerio de educacion Republica de Colombia. (20 de 07 de 2009). minedu. Recuperado el 29 de 10 de 2018, de educacion superior: https://www.mineducacion.gov.co/1621/article-196477.html

Naciones unidas. (01 de julio de 2017). ONU. Recuperado el 29 de 10 de 2018, de impacto academico: https://academicimpact.un.org/es/content/educaci\%C3\%B3n-superior

Navarro, J. (02 de 12 de 2015). Recuperado el 29 de 10 de 2018, de DefinicionABC: https://www.definicionabc.com/?s=Nivel\%20socioecon\%C3\%B3mico

Navarro, J. (20 de 11 de 2015). DefinicionABC. Recuperado el 29 de 10 de 2018, de Educacion superior: https://www.definicionabc.com/?s=Educaci\%C3\%B3n\%20Superior 
NEREYDA G. ARMENTA, C. C. (14 de 05 de 2008). pepsic. Recuperado el 29 de 10 de 2018, de sociedad $\quad$ y http://pepsic.bvsalud.org/pdf/ripsi/v11n1/v11n1a10.pdf

Porto, J. P. (12 de 01 de 2008). Definiciones.de. Obtenido de definicion de estudiante: https://definicion.de/estudiante/

Quintana, C. (3 de 12 de 2017). educacom. Recuperado el 29 de 10 de 2018, de el aspecto economico en los señores univercitarios: https://www.oas.org/dsd/publications/Unit/oea23s/ch09.htm

Tandazo, H. (27 de 10 de 2018). el aspecto socioeconomico en los studiantes univercitarios. 1. (J. Guanoluisa, Entrevistador) Recuperado el 28 de 10 de 2018

Ucha, F. (26 de 07 de 2012). DefinicionABC. Recuperado el 29 de 10 de 2018, de estudiante: https://www.definicionabc.com/?s=estudiante

Venemedia. (27 de 05 de 2018). Conceptodefinicion.de. Recuperado el 24 de 10 de 2018, de definicion.de: https://conceptodefinicion.de/estudiante/

https://ericfochoa.wordpress.com/2012/01/27/economia-popular-y-empleo-en-ecuador/

https://educacion.gob.ec/wpcontent/uploads/downloads/2016/01/Publicaciones/PUB_EstadisticaEducativaVol1 _mar2015.pdf

https://educacion.gob.ec/wpcontent/uploads/downloads/2016/01/Publicaciones/PUB_EstadisticaEducativaVol1 _mar2015.pdf

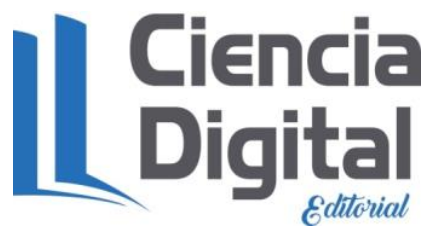


Para citar el artículo indexado.

Carrasco Ruano, Y. (2018). Los estudiantes universitarios y el aspecto socio económico. ConcienciaDigital, 1(2), 6-15. https://doi.org/10.33262/concienciadigital.v1i2.946

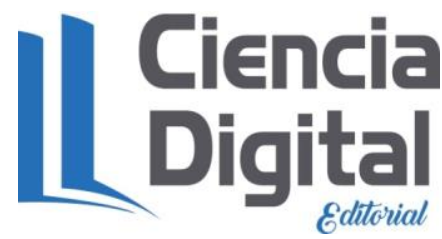

El artículo que se publica es de exclusiva responsabilidad de los autores y no nec6sariamente reflejan el pensamiento de la Revista Conciencia Digital.

El articulo queda en propiedad de la revista y, por tanto, su publicación parcial y/o total en otro medio tiene que ser autorizado por el director de la Revista Conciencia Digital.

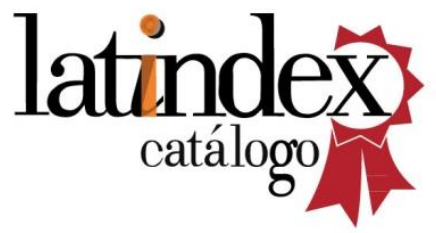

